\title{
A Escola e o Currículo em Tempos de Mobilidade e Conexão: o uso dos computadores portáteis na educação
}

\author{
Jayson Magno da Silva ${ }^{1}$, Maria da Graça Moreira da Silva ${ }^{2}$ \\ ${ }^{1}$ Doutorando em Educação - Programa de Pós-Graduação em Educação: Currículo - \\ Pontifícia Universidade Católica de São Paulo (PUC-SP) \\ Rua Ministro de Godói, 969 - 05015.901 - São Paulo - SP - Brazil \\ ${ }^{2}$ Docente do Programa de Pós-Graduação em Educação: Currículo - Pontifícia \\ Universidade Católica de São Paulo (PUC-SP) \\ jaysonmagno@hotmail.com, graca-moreira@uol.com.br
}

\begin{abstract}
This article, the empirical nature, aims to present a reflection on the use of mobile wireless technologies, especially the laptop, connected to the Internet, in the context of education focusing on curriculum. The study starts from the hypothesis that the integration of mobile technologies for education and curriculum, while devices that enable new ways to communicate, record and represent the thinking, reconfiguring our presence in the culture, in the world and with the world. The methodology is the literature review, taking the lead authors and researchers as a theoretical base. The title of considerations, the study indicates that putting a computer in the hands of each student is not enough to ensure changes in the school and the curriculum, but allows you to see paths to new opportunities to education, with a view to innovation and learning. In short, the integration of mobility and education demand the assumption of the subject of education as authors and rebuilders of the world, and with the connected mobile devices, especially with laptops with Internet access computers, this world embraces the digital world, in that the Internet can mean the whole world.
\end{abstract}

Resumo. O presente artigo, de natureza empírica, objetiva apresentar reflexão sobre o uso das tecnologias móveis sem fio, especialmente, do computador portátil, conectado à Internet, no contexto da educação com foco no currículo escolar. O estudo parte da hipótese de que a integração das tecnologias móveis à educação e ao currículo, enquanto dispositivos que possibilitam novas formas de comunicar, registrar $e$ representar o pensamento, reconfigurando nossa presença na cultura, no mundo e com o mundo. A metodologia empregada é a revisão de literatura, tomando os principais autores e pesquisadores da área enquanto base teórica. A título de considerações, o estudo sinaliza que colocar um computador nas mãos de cada aluno não é suficiente para garantir mudanças na escola e no currículo, mas permite enxergar caminhos para novas oportunidades à educação, com vistas à inovação e à aprendizagem. Em suma, a integração entre a mobilidade e a educação demanda a assunção dos sujeitos da educação enquanto autores e reconstrutores do mundo, e com os dispositivos móveis conectados, sobretudo, com os computadores portáteis com acesso à Internet, esse mundo abarca o mundo digital, o que na Internet pode significa o mundo todo. 


\section{Estabelecendo a conexão}

A escola do século XXI se situa num tempo e espaço sócio histórico no qual a mobilidade tecnológica aliada à conexão da rede mundial está cada vez mais presente em seu cotidiano, de forma ubíqua e pervasiva.

As tecnologias e os dispositivos móveis como notebook, netbook, tocadores de áudio, tablet, telefones celulares inteligentes (smartphones), permitem a conexão móvel à internet e integram mídias convergentes que possibilitam novas formas de comunicar, registrar e representar o pensamento, reconfigurando nossa presença na cultura, no mundo e com o mundo.

Para além da comunicação o uso intensivo das tecnologias nas diversas áreas do conhecimento provoca mudanças no cotidiano - fora e dentro da escola, seja por meio dos novos signos que fluem nas redes conectadas, na construção de novas sociabilidades, na edificação das relações de poder, configurando-se "como uma espécie de modus vivendi, como um processo social que determina as reconfigurações identitárias dos indivíduos" e também dos agentes educacionais. (ZUIN, 2010, p. 967).

A escola está em meio ao debate sobre o uso das tecnologias, suas contradições, seus usos e seus impactos na aprendizagem, no ensino e nas relações pedagógicas. É importante ressaltar que alguns equívocos sobre o uso das tecnologias na escola devem ser esclarecidos: o primeiro é associar o uso com a introdução de dispositivos tecnológicos para motivar o aluno ou para tornar as aulas mais atrativas; o segundo é acreditar que dispor dispositivos tecnológicos móveis e conectados aos alunos poderá provocar a inclusão digital do aluno, do professor e da escola; e o terceiro a ser esclarecido reside na crença de que o uso de tecnologias pode provocar inovações curriculares e, como consequência, provocar mudanças na educação.

Esses equívocos são decorrentes do senso comum e limitam o uso das tecnologias aos aparatos e não às características que imprimem na cultura.

A Pesquisa TIC Educação 2013, realizada pelo Comitê Gestor de Internet no Brasil - CGI (2013a; 2013b), revela que 100\% das escolas públicas do país possuem computadores de mesa e $73 \%$ possuem computadores portáteis. E desses, apenas $6 \%$ estão na sala de aula. O percentual expressivo das máquinas se concentra na área administrativa e em outros espaços como a sala dos professores e o laboratório de informática.

Em relação à conexão à internet, a mesma pesquisa revela que $71 \%$ das escolas públicas brasileiras possuem conexão à internet, embora com velocidade ainda lenta. Em contrapartida, a residência do aluno é apontada como local em que o acesso à internet se dá em $65 \%$ dos casos e apenas $8 \%$ na escola.

Esses dados revelam dois aspectos que causam inquietações: o pouco acesso aos computadores nas salas de aula das escolas públicas nas mãos dos alunos e o descompasso entre o acesso e a conexão dentro e fora da escola. 
Weinberger (2003) conceitua esse momento histórico como a "Era das conexões" pontuando que a conexão se configura como um dos princípios constitucionais da democracia e da economia nas sociedades. Portanto, estar alheado ao acesso à internet e sem estar conectado às redes pode ser interpretado como formas de exclusão digital e social à contemporaneidade.

Duncan Green (2009) diz que surfar pela web se configura um desafio à democratização da internet e à participação em redes colaborativas e sociais como elemento de humanização.

Entretanto, a questão da exclusão não se resume à estar ou não conectado à sociedade do capital que tem como produto previsível a própria exclusão. Nesse cenário, estar conectado é uma questão de direito, uma condição para o exercício da cidadania plena.

Almeida (2009) destaca que é oportuno voltar os olhares às tecnologias digitais para repensá-las no sentido de sua verdadeira democratização, como um direito humano, assim como é direito saber ler, escrever e contar.

Nessa nova cultura, a escola e o currículo não podem estar alheados. Daí, a questão que se coloca é: Como democratizar o acesso às tecnologias digitais móveis conectadas por meio da educação?

\section{Integração entre mobilidade e educação}

Antes de responder a questão é oportuno entender um pouco mais sobre a integração entre mobilidade e educação.

De acordo com Lemos (2008), o conceito de mobilidade é entendido para além do transporte de um dispositivo físico de pequeno porte ou do acesso à informação em qualquer lugar e em qualquer tempo, ou seja, da portabilidade que abarca características da conexão sem fio à internet que oportunizam a mobilidade física e a informacional.

Segundo o autor, há pelo menos três tipos de mobilidade: a mobilidade física/espacial (locomoção, transportes); a mobilidade cognitiva/imaginária (pensamentos, sonhos, religião); a mobilidade virtual/informacional (dispositivos móveis, mídias locativas).

Diante disso, falar sobre mobilidade é considerar duas importantes características ligadas ao conceito: a dimensão tecnológica, que permite a utilização de um dispositivo em diferentes localidades e em movimento e a dimensão de conteúdo, que diz respeito à produção, acesso e difusão da informação, ao lugar onde ela pode ser produzida, onde se encontra, não importado o fato em si, mas a oportunidade de ser distribuída, compartilhada e acessada por inúmeros interessados.

Nas palavras de Lemos (2004), mobilidade são os espaços sociais conectados, definidos pelo uso de interfaces portáteis como os nós da rede... "a transformação das 
interfaces estáticas em interfaces móveis, o que define nossa percepção de espaços digitais". (p. 1).

Para Santaella (2007), a evolução dos computadores, dos dispositivos e das conexões móveis que se comunicam em rede faz com que o cérebro se movimente juntamente com a atividade corporal nas cidades. Desse modo, a cultura da mobilidade passa a ser inerente ao ser humano, agregando questões que vão para além dos objetos em si, envolvendo também os campos político, econômico e social.

Ao falar sobre o contexto da educação, Reinhard et al (2007) pontuam que aprender com mobilidade não é uma ideia nova, mas é uma possibilidade que sempre foi buscada e potencializada com ferramentas como livros, cadernos e outros instrumentos móveis (portáteis) que existem há muito tempo.

Vale destacar que as TMSF possuem peculiaridades que permitem aos usuários diferentes formas de acesso aos conteúdos, produção, compartilhamento e distintas maneiras de organizar as informações, e possibilitam novas oportunidades para a aprendizagem.

As TMSF, sobretudo com a conectividade, possibilitam que a aprendizagem não esteja necessariamente restrita a um local físico no qual estas tecnologias estejam disponíveis, mas sim ao acesso às informações, à interação com outros usuários, à produção e ao compartilhamento de conteúdos. Isso significa que a aprendizagem, que passa a acontecer em qualquer lugar e em qualquer momento, cria outros contextos que permitem aos sujeitos novas formas de interagir e produzir conhecimentos.

Silva (2013) destaca que o conceito de aprendizagem com mobilidade não foca a tecnologia. Para a autora não é possível fazer a leitura da integração entre educação e mobilidade com o mesmo olhar da educação (não móvel) convencional. Conforme pontua Almeida (2009) "a leitura deste mundo não pode ser feita com os mesmos instrumentos de mundos passados". (p.30).

Nesse entender, educação na perspectiva da cultura digital móvel e conectada demanda novos olhares e novos instrumentos de leitura e interpretação do mundo. Portanto, não se trata de interpretar a integração entre educação e mobilidade segundo o componente de deslocamento do humano ou do computador, e sim da leitura crítica da cultura digital móvel conectada e suas possibilidades na educação e potencialidades para mudanças no currículo e na aprendizagem.

Silva e Silva (2014) destacam que o currículo é o próprio acontecer da escola, enquanto prática concreta que se estabelece na intimidade da sala de aula, nos corredores da escola, no laboratório de ciências, nas reuniões entre os diferentes sujeitos e a comunidade, na aula de matemática, na forma de organizar o tempo e o espaço, na cor escolhida para pintar as paredes e etc..

Na compreensão de Almeida (2013) a relevância do uso de TMSF no desenvolvimento do currículo se justifica pelas novas possibilidades de reconfiguração 
da ação pedagógica, de abertura e flexibilidade do currículo, de mobilidade de tempo, espaço e contexto, de construção de novos significados e exercício da autonomia.

Conforme destacado por Silva (2013), cada dispositivo possui peculiaridades e isso também diferencia a forma de acessar os recursos, os conteúdos e de disponibilizar e organizar as informações.

Diante das pesquisas, estudos e reflexões de autores e pesquisadores que foram convidados à interlocução sobre a questão da mobilidade no contexto da educação, retoma-se a questão inicial - como democratizar o acesso às tecnologias digitais móveis conectadas por meio da educação?

Com o propósito de ensaiar uma possível resposta, nas próximas linhas traz-se à tona o retrato de algumas experiências com uso de computadores portáteis com conexão à Internet no contexto da educação.

\section{A tecnologia móvel conectada nas mãos dos sujeitos da educação}

Projetos objetivando introduzir o uso de tecnologias móveis na educação com os computadores portáteis na modalidade 1:1 (um para um), sobretudo no contexto escolar, têm sido desenvolvidos em diversos países do mundo. De acordo com Warschauer e Ames (2010) há algumas experiências sendo feitas no Paraguai, Peru, Nação de Nieu, México, Ruanda, Estados Unidos, Uruguai e outros.

A ideia de cada criança ter seu computador - One Laptop per Child (OLPC) -, conforme descreve Almeida e Valente (2011), nasceu por meio da proposta de Alan Kay em 1968, quando ao visitar Seymour Papert no Massachussetts Institute of Technology (MIT), ficou impressionado com as crianças que resolviam problemas complexos de matemática utilizando o computador.

Diante disso, Kay passou a entender a importância de cada criança ter sua máquina, e a idealizar um computador portátil como meio para expressar e comunicar o que as crianças estavam pensando. Esse computador foi materializado em 1972 como o Dynabook, desenvolvido pelo Learning Research Group (LRG), criado por ele como parte do laboratório Xerox Park. (KAY, 2002). Nos anos 90 a proposta da OLPC foi impulsionada por Nicholas Negroponte, com adesão de diversos países.

De acordo com Valente (2011), nos Estados Unidos (EUA) o uso de computadores portáteis nas escolas teve início em 1997 com o Programa Anytime, Anywhere Learning, o qual levou em cinco anos cerca de mil equipamentos para as escolas, entre elas as da rede pública, e a partir de 2011 teve início a implantação do uso de computadores portáteis comerciais na situação 1:1.

Já no Uruguai, conforme destacado por Silva (2014), a política pública Plano Ceibal (Conectividad Educativa de Informática Básica para el Aprendizagen en Línea), que se constitui em uma ação que se desenvolve com uso de computadores portáteis, tablets e outras tecnologias nas escolas das redes pública e privada de todo o país. 
Rodriguez e Teliz (2011) lembram que a experiência piloto do Plano Ceibal com os computadores portáteis iniciou-se na cidade de Cardal, departamento da Florida, na República Oriental do Uruguai, nos primeiros meses do ano 2007. Em 2009, momento em que a ação chega a capital de Montevidéu, culminou na universalização do acesso gratuito às máquinas disponibilizadas nas mãos de todos os alunos, alunas e docentes, das 2064 (duas mil e sessenta e quatro) escolas do país.

Documento publicado pela UNESCO (2009) sinaliza que o Plano Ceibal constitui-se uma política de uso de tecnologias na educação que possui três dimensões a educacional, a social e a tecnológica -, e seu objetivo é melhorar a qualidade da educação mediante a integração das Tecnologias Digitais da Informação e Comunicação (TDIC) no contexto da sala de aula, envolvendo crianças, escola e família, oportunizando igualdade de condições para todas as crianças e a democratização do conhecimento.

Nas palavras de Pittaluga e Rivoir (2012), o uso dos computadores portáteis, com a experiência Ceibal, mostra benefícios na aprendizagem das crianças, e a natureza ubíqua da tecnologia utilizada se traduz no encontro de crianças com as máquinas em locais públicos com acesso à internet. As análises das pesquisadoras destacam dimensões importantes a serem observadas e exploradas com o uso das TMSF nos contextos de educação, e que merecem pesquisas mais detalhadas - a aprendizagem e a ubiquidade.

Já no Brasil a ideia da OLPC foi recontextualizada para Um Computador por Aluno (UCA), o qual prevê o uso de um dispositivo móvel conectado à internet na mão de cada aluno, e sua implantação tinha como objetivo a inclusão digital e adensamento da cadeia produtiva comercial brasileira.

O projeto está em desenvolvimento no país desde 2007 com o experimento inicial em cinco escolas ${ }^{3}$ em diferentes estados acompanhados por educadores e pesquisadores (fase 1) e posteriormente com o piloto em pouco mais de trezentas escolas públicas brasileiras (fase 2). As escolas públicas selecionadas nas fases 1 e 2 receberam computadores portáteis de baixo custo, objetivando, principalmente a inclusão digital e social da comunidade escolar. (UCA, 2010).

Segundo pesquisadores da área, tais como, Almeida e Prado (2011), Almeida e Valente (2011), M. Almeida (2013), a chegada dos computadores portáteis nas escolas integrantes do Projeto UCA envolveu a reorganização da infraestrutura tecnológica com conexão sem fio, os espaços da sala de aula, o processo de formação de professores e gestores, as relações entre alunos, docentes, outros profissionais do ensino e a comunidade, com traços marcantes de mudanças no currículo devido ao uso dos recursos disponíveis nos computadores e da conexão à internet.

\footnotetext{
${ }^{3}$ Uma escola da cidade de Porto Alegre (Rio Grande do Sul) e outra de São Paulo, uma escola de Piraí (Rio de Janeiro) e outra em Palmas (Tocantins) que utilizam o ClassMate, e uma escola de Brasília que lança mão do uso do Laptop Mobilis.
} 
O Projeto UCA reorientou o foco do olhar dos educadores para o papel da escola como integrante da cultura digital móvel, as possíveis alterações em sua ecologia, os tempos e espaços para a aprendizagem e o desenvolvimento do currículo com a participação dos sujeitos da educação.

Assim, esse currículo remodelado com uso de TMSF conectadas passa a ser construído em diferentes tempos e espaços, criando diferentes contextos de aprendizagem, envolvendo alunos, tecnologia e conhecimento, enquanto uma construção marcadamente cultural, social e política.

Almeida e Valente (2011), Almeida (2013), Valente (2013; 2014) ao pautarem os resultados que se destacam na literatura e pesquisas em âmbitos nacional e internacional quanto ao uso do computador portátil no contexto escolar no modelo 1:1, apontam que alunos envolvidos nesse tipo de ação escrevem melhor do que alunos que basicamente utilizam apenas lápis e papel; têm melhor compreensão da informação; apresentam melhor desempenho no trabalho em grupo e na colaboração com os pares; demonstram melhor uso das tecnologias em atividades na escola e fora dela, e tem acesso mais rápido e imediato à informação, o que contribui com as discussões no lócus da sala de aula, assim como em outros espaços dentro e fora da escola, inclusive no mundo digital.

No âmbito pedagógico, a introdução de computadores nas mãos dos alunos e professores descortina inúmeras possibilidades para o processo de ensino e aprendizagem e para o currículo, porém essa utilização deverá ser precedida pela formação dos professores e gestores escolares, além de outros componentes que compõe a prática pedagógica, como: planejamento, desenvolvimento de atividades e avaliação.

Corroborando com essa ideia Costa et al (2012) aponta que o processo de formação dos professores não pode estar centrado na aprendizagem do uso das tecnologias, mas segundo Silva (2013), deve ir mais além, englobando a apropriação e inserção dos educadores na Era digital ou cultura digital (ressalta-se, móvel e conectada), o que significa para a escola uma oportunidade de se enxergar como partícipe deste mundo digital e atuar como leitora crítica e autora desse mundo.

De acordo com Silva (2013), a apropriação do uso de computadores portáteis no modelo 1:1, e seu uso inovador pode se iniciar, mas não se limita ao uso desses dispositivos na escola e não se encerra com a descoberta de novos contextos de aprendizagem, e sim envolve, nesse processo, a consciência do papel da escola como integrante da cultura digital móvel e conectada, e as possíveis mudanças em sua ecologia e desenvolvimento do currículo.

Sendo assim, as tecnologias móveis conectadas podem redesenhar o contexto da escola com novas formas de comunicar, de informar, de gerenciar, de produzir conhecimentos, de ensinar e de aprender. 
Ao levar em consideração os contextos de aprendizagem, a educação com mobilidade permite aos sujeitos da educação - gestores, professor, aluno e toda a comunidade escolar - se assumirem enquanto atores e autores de sua própria história, que podem ler e interpretar o mundo, lançando sua voz (palavra) nele, com ele e sobre ele.

Conforme Silva (2011) e Silva e Silva (2013; 2014), lançar a voz no mundo, com o mundo e sobre o mundo, significa uma oportunidade de autoria, de participação e de empoderamento (empowerment), que as TDIC podem favorecer no sentido da emancipação dos sujeitos que fazem e refazem a escola, tanto na concretude do seu chão como no mundo digital e portanto, no mundo todo.

Ler criticamente o mundo, intervir nele, lançando sua voz no espaço digital com o uso de computadores portáteis com conexão à Internet, pode ser um passo importante no processo de mudanças e inovação na escola e no currículo.

\section{4. (In) Conclusão}

Com fundamento na bibliografia utilizada no presente artigo, é possível ensaiar uma breve análise que pode trazer contribuições para os próximos capítulos do livro vivo que os pesquisadores ao redor do mundo vêm escrevendo sobre a temática que pauta o uso das tecnologias móveis conectadas na área da educação.

A análise encetada permitiu entender que escola está diante dos desafios contemporâneos provocados pela era das conexões - ou cultura digital, em tempos de mobilidade, no qual os avanços tecnológicos caminham em aliança com mudanças, que se manifestam na cultura, economia, política, sociedade e não deixam de fora o interior da sala de aula, o contexto da escola e o currículo.

Juntamente com essas mudanças, alinham-se o compromisso com a democracia e a justiça social, traduzida por meio da prática de acesso à tecnologia. Mudanças que não se realizam pelas tecnologias, mas também não se operam sem elas. São condições e não suas causas.

Colocar um computador nas mãos de cada aluno não é suficiente para garantir mudanças na escola e no currículo, mas abarca certo grau de complexidade, pois deve preceder mudanças na visão de educação, de currículo e de organização escolar e na formação de educadores. Essa mudança, que pressupõe inovação, deve vir acompanhada do movimento de agregar valor à atividade que o aluno e o professor realizam, o que implica na integração das TDIC com o currículo e, portanto, com a própria vida da escola.

A mobilidade integrada à educação demanda a assunção dos sujeitos da educação como autores e reconstrutores do mundo, no mundo e com o mundo. Envolve o processo de leitura e interpretação crítica desse mundo e o exercício democrático de conferir voz a esses sujeitos para que possam lançar suas palavras sobre o mundo. 
Nesse contexto, reconfiguram-se os tempos e espaços da escola e do currículo. A aprendizagem se torna favorecida pela mobilidade, ampliando o repertório e os conhecimentos aprendidos e apreendidos por meio das tecnologias móveis conectadas nas mãos dos sujeitos da educação.

Em virtude de tudo o que foi mencionado é-se levado a acreditar que o tema educação versus currículo em tempos de mobilidade conectada é uma área de estudos que merece ser profundamente investigada e disseminada.

\section{Referências}

ALMEIDA, F. J. (2009) “Paulo Freire”, Coleção Folha explica, n.81, São Paulo, Publifolha.

ALMEIDA, M. E.B. (2013) O computador portáteil e a inovação educativa: das intenções à realidade, In: ALMEIDA, M. E. B. e PAULO DIAS, B. D. S. (Orgs.) "Cenários de inovação para a educação na sociedade digital", São Paulo, Edições Loyola.

ALMEIDA, M. E. B. e PRADO, M. E. B. B. (Orgs.) (2011) “O computador portátil na escola: mudanças e desafios nos processos de ensino e aprendizagem”, São Paulo, Avercamp.

ALMEIDA, M. E. B. e SILVA, M. G. M. (2011) Currículo, tecnologia e cultura digital: espaços e tempos de web currículo, In: "Revista e-curriculum", v. 7, n. 1. Disponível em $<$ http://revistas.pucsp.br/index.php/curriculum/article/view/5676>. Acesso em 19/julho/2011.

ALMEIDA, M. E. B. e VALENTE, J. A. (2011) "Currículo e tecnologias: trajetórias convergentes ou divergentes?", Coleção questões fundamentais da educação, v. 10, São Paulo, Paulus.

CÂMARA DOS DEPUTADOS. (2008) "Um computador por aluno: a experiência brasileira", Brasília, Câmara dos Deputados, Coordenação de Publicações.

COMITÊ GESTOR DE INTERNET NO BRASIL. (2013a) "Pesquisa TIC Educação 2013: pesquisa sobre o uso das tecnologias de informação e comunicação nas escolas brasileiras apresentação dos principais resultados", São Paulo, CGI. Disponível em: < http://cetic.br/media/analises/tic-educacao-apresentacao-2013.pdf >. Acesso em 10/09/2014.

. (2013b) "Tecnologias e educação: o uso da internet por alunos brasileiros de ensino fundamental e médio", ano 5, n.2, São Paulo, CGI. Disponível em: $<$ http://cetic.br/media/docs/publicacoes/6/Panorama\%20Setorial\%20AGOSTO\%202013 FI NAL.pdf $>$. Acesso em 10/09/2014.

COSTA, F. A., RODRIGUEZ, C., CRUZ, E. e FRADÃO, S. (2012) "Repensar as TIC na educação: o professor como agente transformador", Lisboa, Santillana.

GREEN, D. (2009) "Da Pobreza ao Poder: como cidadãos ativos e estados efetivos podem mudar o mundo", São Paulo, Cortez.

KAY, A. (2002) "The Dynabook revisited", A conversation with Allan Kay. Disponível em <http://www.squeakland.org/content/articles/attach/dybabook_revisited.pdf $>$. Acesso em $11 / 10 / 2013$.

LEMOS, A. (2004) Cibercultura e mobilidade: a era da conexão, In: LEÃO, Lucia (Org.) "Derivas: cartografias do ciberespaço", São Paulo, Annablume, pp.17-44.

(2008) Mídias locativas e territórios informacionais, In: SANTAELLA, L. e ARANTES, P. (Orgs.) "Estéticas Tecnológicas: novos modos de sentir", São Paulo, EDUC, pp. 207-30. 
NEGROPONTE, N. (1995) “Ser digital”, México, DF, Océano.

PITTALUGA, L. e RIVOIR, A. (2012) One Laptop per Child and Bridging the Digital Divide: The Case of Plan CEIBAL in Uruguay, In: "Information Technologies \& International Development", v.8, n.4, Los Angeles, University of Southern California, pp. 145-59.

RODRIGUEZ, E. e TELIZ, F. (2011) Implementación Del plan ceibal em Uruguay: revisión de investigaciones y desafios de mejora. In: "Revista Iberoamericana de Evaluación Educativa", v.4, n.2, Madrid, Universidad Autonoma de Madrid, pp. 55-71.

REINHARD, N., SACCOL, A. Z., SCHLEMMER, E., BARBOSA, J. e KRISTOFFERSEN, S. (2007) "Aprendizagem com mobilidade no contexto organizacional". Disponível em: <http://www.inf.unisinos.br/ mobilab/>. Consulta em 02.02.2014.

SANTAELLA, L. (2007) “Linguagens Líquidas na Era da Mobilidade”, São Paulo, Paulus.

SILVA, J. M. (2011) "O som da integração das tecnologias digitais da informação e comunicação ao currículo: a rádio na internet - voz, poder e aprendizagem", Dissertação (Mestrado em Educação) - Programa de Pós-Graduação em Educação: Currículo da Pontifícia Universidade Católica de São Paulo, São Paulo, PUC-SP.

. (No Prelo). (2014) "Uma investigação exploratória do estado da arte da pesquisa sobre o Plano Ceibal do Uruguai”, Programa de Pós-Graduação em Educação: Currículo da Pontifícia Universidade Católica de São Paulo, São Paulo, PUC-SP.

. e SILVA, M. G. M. (2013) Autoria no mundo digital: o currículo na voz dos sujeitos da aprendizagem, In: "Revista de Educação PUC-Campinas", v.18, n.2, Pontifícia Universidade Católica de Campinas, Centro de Ciência Humanas e Sociais Aplicadas, Programa de Pós-Graduação em Educação, Campinas, PUC-Campinas, pp.191-9. Disponível em: <http://periodicos.puc-campinas.edu.br/seer/index.php/reveducacao/article/view/2028>. Consulta em 14/10/2013.

. (2014) Integração das tecnologias digitais ao currículo: a rádio na internet e a emergência de web currículo, In: ALMEIDA, M. E. B., ALVES, R. M. e LEMOS, S. D. V. (Orgs.) "Web Currículo: aprendizagem, pesquisa e conhecimento com uso de tecnologias digitais", Rio de Janeiro, Letra Capital, pp. 71-81.

SILVA, M. G. M. (2013) Mobilidade e construção do currículo na cultura digital, In: ALMEIDA, M. E. B. e PAULO DIAS, B. D. S. (Orgs.) "Cenários de inovação para a educação na sociedade digital”, São Paulo, Edições Loyola, pp.123-35.

UCA. (2010) "Um Computador por Aluno", Brasília, DF, Ministério da Educação. Disponível em: <www.uca.gov.br>. Consulta em 16.07.2014.

UNESCO. (2009) Organizacion de las Naciones Unidas para La Educación, La Ciencia e La Cultura, "En el caminho del Plan Ceibal: referencias para padres y educadores", UNESCO, Anep, Montevideo.

VALENTE, J. A. (2011) Um computador para cada aluno: promessas e resultados educacionais efetivos, In: Almeida, M.E.B. e Prado, M.E.B.B. (Orgs.) "O computador portátil na escola: mudanças e desafios nos processos de ensino e aprendizagem”, São Paulo, Avercamp, pp.2033.

WARSCHAUER, M. e AMES, M. (2010) Can one laptop per child save the world's poor?, "Journal of International Affairs", v.64, n.1, New York, Columbia University, pp.33-51.

WEINBERGER, D. (2003) "Why Open Spectrum Matters. The end of the broadcast nation". Disponível em <http://www.evident.com>. Acesso em 19/Julho/2011.

ZUIN, A. S. (2010) O Plano Nacional de Educação e as tecnologias da informação e comunicação, In: "Educ. Soc.”, Campinas, v. 31, n. 112, p. 961-980, jul.-set. 